\title{
On the Structure of Complex Clifford Algebra
}

\author{
F. Brackx, H. De Schepper and V. Souček
}

\begin{abstract}
The structure of a complex Clifford algebra is studied by direct sum decompositions into eigenspaces of specific linear operators.
\end{abstract}

Mathematics Subject Classification (2000). 11E88, 15A66, 15A75, 30G35.

Keywords. complex Clifford algebra, spinor space.

\section{Introduction}

Usually Clifford analysis is understood to be the study of the solutions of the Dirac equation for functions defined on Euclidean vector space $\mathbb{R}^{m}$ and taking values in the corresponding Clifford algebra $\mathbb{R}_{0, m}$. In this way it offers a proper analogue to the Cauchy-Riemann equations for holomorphic functions in the complex plane. For a thorough study of the so-called monogenic functions of Clifford analysis we refer to the standard textbooks $[5,16,17,18]$.

The symmetry group of the Dirac equation is either $\mathrm{SO}(m)$ or $\operatorname{Spin}(m)$, according to the definition of the group action on the values taken by the functions under consideration. If these values are in the Clifford algebra with left multiplication, the symmetry group is $\operatorname{Spin}(m)$, which then usually is realized inside the Clifford algebra. In the case of functions with values in the Clifford algebra with both side action, it is more natural to identify the Dirac equation with the equation $\left(d+d^{*}\right) f=0$, and to identify the space of values, in casu the Clifford algebra, as a vector space, with the Grassmann algebra of $\mathbb{R}^{m}$. This Grassmann algebra may then be decomposed into the direct sum of its homogeneous parts, which is a decomposition into irreducible parts under the action of $\mathrm{SO}(m)$. In this framework it was shown (see $[14,19,20]$ ) that, on the polynomial level, the space of monogenic functions can be split into a direct sum of solutions of the Hodge-de Rham equations for homogeneous differential forms. This entails a finer structure of the space of monogenic functions, which manifests itself explicitly in a finer form of the corresponding Fischer decomposition (see [15]). 
When the dimension is taken to be even $(m=2 n)$, one can make the framework of Clifford analysis closer to complex analysis by introducing on $\mathbb{R}^{2 n}$ a complex structure $J$. The symmetry group then reduces to the subgroup $\mathrm{U}(n) \subset$ $\mathrm{SO}(2 n)$ preserving the chosen complex structure $J$. This is the basic setting for so-called Hermitean Clifford analysis, which recently has emerged as a new branch of Clifford analysis, offering yet a refinement of the Euclidean case. The functions studied are defined in open regions of $\mathbb{C}^{n}$ and take their values in the complex Clifford algebra $\mathbb{C}_{2 n}$. More particularly Hermitean Clifford Analysis focusses on the simultaneous null solutions, called Hermitean (or $\mathrm{h}-$ ) monogenic functions, of two Hermitean Dirac operators $\partial_{z}$ and $\partial_{z^{\dagger}}$. A systematic development of this function theory, including the invariance properties with respect to the underlying Lie groups and Lie algebras, is still in full progress, see e.g. [10, 1, 2, 7, 9, 3, 4, 24, 13].

Part of this program is also the study of the finer structure of the space of monogenic functions induced by the choice of the complex structure $J$. As a first step towards that goal in [11], a scheme for the translation of spaces and operators between the language of complex Clifford algebra and the language of complex differential forms was established. In fact this is the complex analogue of the translation in the Euclidean situation (see [6]). In the underlying paper we focus on the structure of the complex Clifford algebra in which the monogenic functions take their values. Let us explain this in some more detail.

When studying the Dirac equation for functions with values in a Clifford algebra, it is well-known that the Clifford algebra splits into the direct sum of isomorphic copies of the basic spinor representation. Accordingly, the system of equations will split into a number of independent, however mutually equivalent, subsystems of equations, whence, without loss of generality, the study may be restricted to functions with values in the space of spinors (or half-spinors in even dimension). In the standard situation, this space of values cannot be split further since they are already irreducible under the (left) action of the $\operatorname{Spin}(m)$ group. However, by the introduction of the complex structure $J$, the symmetry group is reduced, as explained above, and spinor space decomposes further into smaller pieces. If spinor space is realized in a standard way as the Grassmann algebra over the maximal isotropic subspace in $\mathbb{C}^{2 n}$, then this splitting is just the splitting into homogeneous components (for details see, e.g., [2]).

The reduction of the symmetry group to $\mathrm{U}(n)$ gives to the (complex) Clifford algebra in even dimension $m=2 n$ a quite fine and complicated structure. A nice description of a 'coarser' structure of the complex Clifford algebra induced by the choice of $J$, can be found in the seminal paper [21] by Michelsohn. This basic information is reviewed here and complemented by a discussion of a finer structure based on a full study of the decomposition of the Clifford algebra into a sum of spinor spaces induced by the left, respectively the right action, and a description of the relation of this finer decomposition to the coarser one used by Michelsohn. 
The paper is structured as follows. After some preliminaries concerning the complex Clifford algebra (Section 1), the left and right decomposition into isomorphic copies of spinor space is discussed in Section 2; finer blocks $\mathbb{U}^{(s, v)}$ are also introduced here. Two operators $\mathcal{J}$ and $\mathcal{K}$, introduced by Michelsohn are recalled, in our notation, in Sections 3 and 4 and the relation between both is discussed in Section 5. In particular, we are describing carefully the left, respectively right action on the Clifford algebra by elements in the maximal isotropic subspaces of $\mathbb{C}^{2 n}$. This is the key information for a definition for certain elliptic complexes in the Michelsohn paper and it will be the key information as well for the study of a finer structure on the space of monogenic functions, i.e. the next step in the study of solutions in the Hermitean Clifford context in the forthcoming paper [8].

\section{Complex Clifford algebra}

A Clifford algebra is a normed associative algebra, but not a division algebra; special cases are e.g. the complex numbers and the quaternions. Clifford numbers may be split into Grassmann blades, the ones of homogeneity degree 1 being identified with the usual vectors. The geometric product of vectors is a combination of the commutative scalar or inner product and the anti-commutative wedge or outer product. The scalars may be real or complex, and in this paper we consider a complex Clifford algebra constructed over a quadratic space of even dimension. Our aim is to obtain decompositions of the complex Clifford algebra into subspaces allowing for exact sequences under the multiplicative action of the so-called Witt basis vectors. In an afterword we briefly sketch the analysis background and motivation for this research.

The construction of the universal real Clifford algebra is well-known; for an in-depth study we refer the reader to e.g. [23]. Here we restrict ourselves to a schematic approach. Let $\mathbb{R}^{0, m}$ be the real vector space $\mathbb{R}^{m}(m \geq 1)$ endowed with a non-degenerate symmetric bilinear form $\mathcal{B}$ of signature $(0, m)$, and let $\left(e_{1}, \ldots, e_{m}\right)$ be an associated orthonormal basis, i.e.

$$
\mathcal{B}\left(e_{i}, e_{j}\right)=\left\{\begin{aligned}
-1 & \text { if } \quad i=j \\
0 & \text { if } \quad i \neq j
\end{aligned} \quad(1 \leq i, j \leq m)\right.
$$

then the anti-Euclidean metric on $\mathbb{R}^{0, m}$ is induced by the scalar product

$$
\left\langle e_{i}, e_{j}\right\rangle=-\mathcal{B}\left(e_{i}, e_{j}\right)=\delta_{i j}, \quad 1 \leq i, j \leq m
$$

We first introduce the anti-symmetric outer product by the rules

$$
\begin{aligned}
e_{i} \wedge e_{i} & =0, \quad 1 \leq i \leq m \\
e_{i} \wedge e_{j}+e_{j} \wedge e_{i} & =0, \quad 1 \leq i \neq j \leq m
\end{aligned}
$$

and for each $A=\left\{i_{1}, i_{2}, \ldots, i_{r}\right\} \subset M=\{1, \ldots, m\}$, with $1 \leq i_{1}<i_{2}<\ldots<i_{r} \leq m$, i.e. ordered in the natural way, we put $e_{A}=e_{i_{1}} \wedge e_{i_{2}} \wedge \ldots \wedge e_{i_{r}}$, while $e_{\emptyset}=1$. 
Then for $r=0,1, \ldots, m$, the set $\left\{e_{A}: A \subset M\right.$ and $\left.|A|=r\right\}$ is a basis for the space $\mathbb{R}_{0, m}^{r}$ of so-called r-vectors. Next, we introduce the inner product

$$
e_{i} \cdot e_{j}=-\left\langle e_{i}, e_{j}\right\rangle=\mathcal{B}\left(e_{i}, e_{j}\right)=-\delta_{i j}, \quad 1 \leq i, j \leq m
$$

leading to the so-called geometric product in the Clifford algebra:

$$
e_{i} e_{j}=e_{i} \bullet e_{j}+e_{i} \wedge e_{j}, \quad 1 \leq i, j \leq m
$$

The respective definitions of the inner, the outer and the geometric product are then extended to $r$-vectors as follows: for the inner product, we have

$$
e_{j} \bullet e_{A}=e_{j} \bullet\left(e_{i_{1}} \wedge \ldots \wedge e_{i_{r}}\right)=\sum_{k=1}^{r}(-1)^{k} \delta_{j i_{k}} e_{A \backslash\left\{i_{k}\right\}}
$$

with

$$
e_{A \backslash\left\{i_{k}\right\}}=e_{i_{1}} \wedge \ldots \wedge e_{i_{k-1}} \wedge\left[e_{i_{k}} \wedge\right] e_{i_{k+1}} \wedge \ldots \wedge e_{i_{r}}
$$

while for the outer product

$$
\left\{\begin{array}{rlrl}
e_{j} \wedge e_{A} & =e_{j} \wedge\left(e_{i_{1}} \wedge \ldots \wedge e_{i_{r}}\right)=e_{j} \wedge e_{i_{1}} \wedge \ldots \wedge e_{i_{r}}, & & \text { if } j \notin A \\
e_{j} \wedge e_{A}=0, & & \text { if } j \in A
\end{array}\right.
$$

and finally, for the geometric product (or product for short)

$$
e_{j} e_{A}=e_{j} \cdot e_{A}+e_{j} \wedge e_{A}
$$

Finally, these definitions are linearly extended to the whole of the Clifford algebra $\mathbb{R}_{0, m}$, which is the associative algebra $\mathbb{R}_{0, m}=\bigoplus_{r=0}^{m} \mathbb{R}_{0, m}^{r}$. If $[\cdot]_{r}: \mathbb{R}_{0, m} \rightarrow \mathbb{R}_{0, m}^{r}$ denotes the projection operator from $\mathbb{R}_{0, m}$ onto $\mathbb{R}_{0, m}^{r}$, then each Clifford number $a \in \mathbb{R}_{0, m}$ may be written as $a=\sum_{r=0}^{m}[a]_{r}$. Note that in particular for a 1-vector $u$ and an $r$-vector $v_{r}$, one has

$$
u v_{r}=u \cdot v_{r}+u \wedge v_{r}
$$

with

$$
\begin{aligned}
& u \bullet v_{r}=\left[u v_{r}\right]_{r-1}=\frac{1}{2}\left(u v_{r}-(-1)^{r} v_{r} u\right) \\
& u \wedge v_{r}=\left[u v_{r}\right]_{r+1}=\frac{1}{2}\left(u v_{r}+(-1)^{r} v_{r} u\right)
\end{aligned}
$$

Usually $\mathbb{R}$ and $\mathbb{R}^{m}$ are identified with $\mathbb{R}_{0, m}^{0}$ and $\mathbb{R}_{0, m}^{1}$ respectively.

When allowing for complex coefficients the same generators $\left(e_{1}, \ldots, e_{m}\right)$ produce the complex Clifford algebra $\mathbb{C}_{m}$, which is the complexification of the real Clifford algebra $\mathbb{R}_{0, m}$, i.e. $\mathbb{C}_{m}=\mathbb{R}_{0, m} \oplus i \mathbb{R}_{0, m}$. Clearly, in a complex Clifford algebra no signature is involved, since each basis vector $e_{j}$ may be multiplied by the imaginary unit $i$ in this way changing the sign of its square. Any complex Clifford number $\lambda \in \mathbb{C}_{m}$ may be written as $\lambda=a+i b, a, b \in \mathbb{R}_{0, m}$, an observation leading to the definition of the Hermitean conjugation $\lambda^{\dagger}=(a+i b)^{\dagger}=\bar{a}-i \bar{b}$, where the bar notation stands for the usual Clifford conjugation in $\mathbb{R}_{0, m}$, i.e. the main 
anti-involution for which $\bar{e}_{j}=-e_{j}, j=1, \ldots, m$. This Hermitean conjugation also leads to a Hermitean inner product and its associated norm on $\mathbb{C}_{m}$ given by

$$
\begin{gathered}
(\lambda, \mu)=\left[\lambda^{\dagger} \mu\right]_{0}, \quad \lambda, \mu \in \mathbb{C}_{m} \\
|\lambda|=\sqrt{\left[\lambda^{\dagger} \lambda\right]_{0}}=\left(\sum_{A}\left|\lambda_{A}\right|^{2}\right)^{1 / 2}, \quad \lambda \in \mathbb{C}_{m}
\end{gathered}
$$

An elegant way of introducing the complex Clifford algebra consists in considering a so-called complex structure, i.e. a specific $\mathrm{SO}(m ; \mathbb{R})$-element $J$ for which $J^{2}=-\mathbf{1}$ (see e.g. $[1,2]$ ). As the introduction of such a complex structure forces the dimension to be even, we put $m=2 n$. More particularly we choose $J$ to act upon the generators $\left(e_{1}, \ldots, e_{n}, e_{n+1}, \ldots, e_{2 n}\right)$ of the Clifford algebra as $J\left[e_{j}\right]=-e_{n+j}$ and $J\left[e_{n+j}\right]=e_{j}, j=1, \ldots, n$. With $J$ one may associate two projection operators $\frac{1}{2}(\mathbf{1} \pm i J)$ which produce the main objects of the Hermitean setting by acting upon the corresponding ones in the Euclidean framework. First of all, the so-called Witt basis elements $\left(\mathfrak{f}_{j}, \mathfrak{f}_{j}^{\dagger}\right)_{j=1}^{n}$ are obtained through the action of $\pm \frac{1}{2}(\mathbf{1} \pm i J)$ on the Euclidean basis:

$$
\begin{aligned}
\mathfrak{f}_{j}=\frac{1}{2}(\mathbf{1}+i J)\left[e_{j}\right]=\frac{1}{2}\left(e_{j}-i e_{n+j}\right), \quad j=1, \ldots, n \\
\mathfrak{f}_{j}^{\dagger}=-\frac{1}{2}(\mathbf{1}-i J)\left[e_{j}\right]=-\frac{1}{2}\left(e_{j}+i e_{n+j}\right), \quad j=1, \ldots, n
\end{aligned}
$$

The Witt basis elements satisfy the Grassmann identities

$$
\mathfrak{f}_{j} \mathfrak{f}_{k}+\mathfrak{f}_{k} \mathfrak{f}_{j}=\mathfrak{f}_{j}^{\dagger} \mathfrak{f}_{k}^{\dagger}+\mathfrak{f}_{k}^{\dagger} \mathfrak{f}_{j}^{\dagger}=0, \quad j, k=1, \ldots, n
$$

including their isotropy: $\mathfrak{f}_{j}^{2}=\mathfrak{f}_{j}^{\dagger^{2}}=0, j=1, \ldots, n$, as well as the duality identities

$$
\mathfrak{f}_{j} \mathfrak{f}_{k}^{\dagger}+\mathfrak{f}_{k}^{\dagger} \mathfrak{f}_{j}=\delta_{j k}, j, k=1, \ldots, n
$$

The Witt basis of the complex Clifford algebra $\mathbb{C}_{2 n}$ is then obtained, similarly to the basis of the real Clifford algebra, by taking the $2^{2 n}$ possible products of Witt basis vectors: $\left(1, \mathfrak{f}_{1}^{\dagger}, \mathfrak{f}_{1}, \ldots, \mathfrak{f}_{1}^{\dagger} \mathfrak{f}_{2}^{\dagger}, \ldots, \mathfrak{f}_{1}^{\dagger} \cdots \mathfrak{f}_{n}^{\dagger} \mathfrak{f}_{1} \cdots \mathfrak{f}_{n}\right)$. More in detail we have, see also [10]:

$$
\begin{aligned}
\mathfrak{f}_{j} \bullet \mathfrak{f}_{k} & =\mathfrak{f}_{j}^{\dagger} \bullet \mathfrak{f}_{k}^{\dagger}=0, \\
\mathfrak{f}_{j} \bullet \mathfrak{f}_{k}^{\dagger} & =\mathfrak{f}_{j}^{\dagger} \bullet \mathfrak{f}_{k}=\frac{1}{2} \delta_{j k}, \\
\mathfrak{f}_{j} \wedge \mathfrak{f}_{k} & =-\mathfrak{f}_{k} \wedge \mathfrak{f}_{j}, \\
\mathfrak{f}_{j}^{\dagger} \wedge \mathfrak{f}_{k}^{\dagger} & =-\mathfrak{f}_{k}^{\dagger} \wedge \mathfrak{f}_{j}^{\dagger}, \\
\mathfrak{f}_{j} \wedge \mathfrak{f}_{k}^{\dagger} & =-\mathfrak{f}_{k}^{\dagger} \wedge \mathfrak{f}_{j},
\end{aligned}
$$$$
j, k=1, \ldots, n
$$$$
j, k=1, \ldots, n
$$$$
j, k=1, \ldots, n
$$$$
j, k=1, \ldots, n
$$$$
j, k=1, \ldots, n
$$

eventually yielding

$$
\begin{array}{rlrl}
\mathfrak{f}_{j} \mathfrak{f}_{k} & =\mathfrak{f}_{j} \bullet \mathfrak{f}_{k}+\mathfrak{f}_{j} \wedge \mathfrak{f}_{k}=\mathfrak{f}_{j} \wedge \mathfrak{f}_{k}, & j, k=1, \ldots, n \\
\mathfrak{f}_{j}^{\dagger} \mathfrak{f}_{k}^{\dagger}=\mathfrak{f}_{j}^{\dagger} \bullet \mathfrak{f}_{k}^{\dagger}+\mathfrak{f}_{j}^{\dagger} \wedge \mathfrak{f}_{k}^{\dagger}=\mathfrak{f}_{j}^{\dagger} \wedge \mathfrak{f}_{k}^{\dagger}, & j, k=1, \ldots, n \\
\mathfrak{f}_{j} \mathfrak{f}_{k}^{\dagger}=\mathfrak{f}_{j} \bullet \mathfrak{f}_{k}^{\dagger}+\mathfrak{f}_{j} \wedge \mathfrak{f}_{k}^{\dagger}=\frac{1}{2} \delta_{j k}+\mathfrak{f}_{j} \wedge \mathfrak{f}_{k}^{\dagger}, & j, k=1, \ldots, n
\end{array}
$$


This leads to the Grassmann structure of the complex Clifford algebra

$$
\mathbb{C}_{2 n} \cong \bigoplus_{p=0}^{n} \bigoplus_{q=0}^{n} \bigwedge_{2 n}^{p, q}
$$

where

$$
\begin{aligned}
\bigwedge_{2 n}^{p, q}=\operatorname{span}_{\mathbb{C}}\left\{\mathfrak{f}_{j_{1}}^{\dagger} \wedge \ldots \wedge \mathfrak{f}_{j_{p}}^{\dagger} \wedge \mathfrak{f}_{k_{1}} \wedge \ldots \wedge \mathfrak{f}_{k_{q}} \mid j_{1}<j_{2}<\ldots<j_{p},\right. \\
\left.k_{1}<k_{2}<\ldots<k_{q}\right\}
\end{aligned}
$$

In this way the complex Clifford algebra $\mathbb{C}_{2 n}$ is partitioned into a "square" of $(n+1)^{2}$ subspaces $\bigwedge_{2 n}^{p, q}$, see Figure 1 for the case $n=2$.

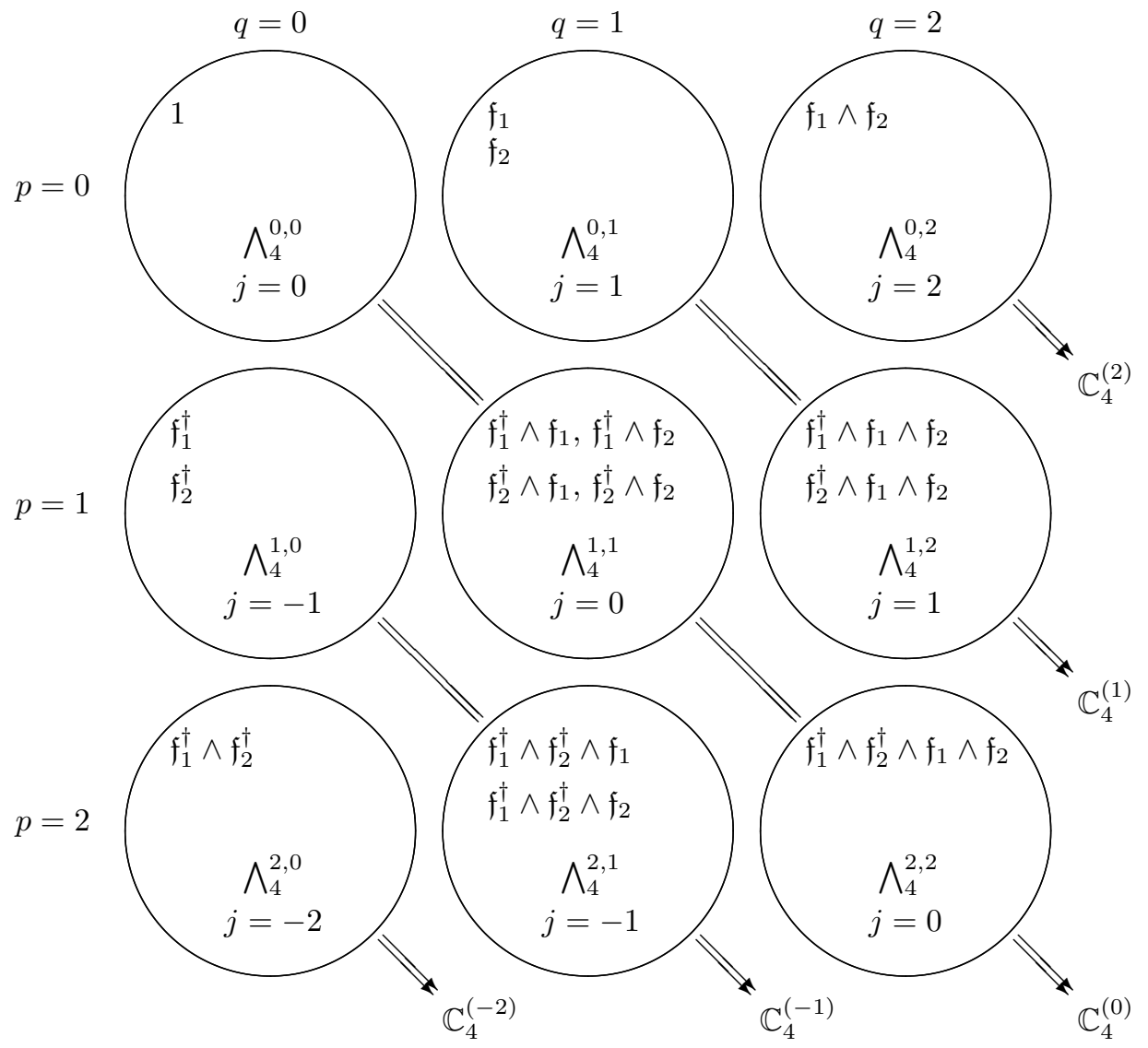

FIGURE 1. Grassmann splitting of the complex Clifford algebra $\mathbb{C}_{4}$ 
This "square" is particularly suited for illustrating the action of the isotropic multiplication operators $\mathfrak{f}_{j} \wedge, \mathfrak{f}_{j} \bullet, \mathfrak{f}_{j}^{\dagger} \wedge$ and $\mathfrak{f}_{j}^{\dagger} \bullet,(j=1, \ldots, n)$. The operators $\mathfrak{f}_{j} \bullet$ and $\mathfrak{f}_{j}^{\dagger} \wedge$ act vertically (upwards and downwards respectively), while the operators $\mathfrak{f}_{j} \wedge$ and $\mathfrak{f}^{\dagger} \bullet$ act horizontally (to the right and to the left respectively), leading to the exact sequences

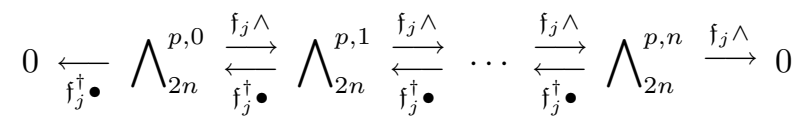

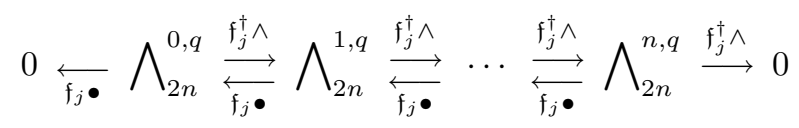

Also note the mirror symmetry with respect to the main diagonal under Hermitean conjugation: $\left(\bigwedge_{2 n}^{p, q}\right)^{\dagger}=\bigwedge_{2 n}^{q, p}$.

\section{Spinor spaces}

Spinor space is defined as a minimal left ideal of the complex Clifford algebra, and realized explicitly by means of a self-adjoint idempotent. A direct construction of the respective realizations of spinor space within the Clifford algebra is obtained by means of the Witt basis. To this end, we start from the duality relation of the Witt basis vectors $\mathfrak{f}_{j} \mathfrak{f}_{j}^{\dagger}+\mathfrak{f}_{j}^{\dagger} \mathfrak{f}_{j}=1, j=1, \ldots, n$, where we put $I_{j}=\mathfrak{f}_{j} \mathfrak{f}_{j}^{\dagger}$ and $K_{j}=\mathfrak{f}_{j}^{\dagger} \mathfrak{f}_{j}, j=1, \ldots, n$, so that it takes the form $1=I_{j}+K_{j}, j=1, \ldots, n$, whence also $1=\prod_{j=1}^{n}\left(I_{j}+K_{j}\right)$. Direct computations then yield the following results.

Lemma 3.1. One has, for all $j, k=1, \ldots, n$, that

(i) $I_{j}$ is a self-adjoint idempotent: $I_{j}^{\dagger}=I_{j}, I_{j}^{2}=I_{j}$;

(ii) $K_{j}$ is a self-adjoint idempotent: $K_{j}^{\dagger}=K_{j}, K_{j}^{2}=K_{j}$;

(iii) $I_{j}$ and $I_{k}$ are commuting: $I_{j} I_{k}=I_{k} I_{j}$;

(iv) $K_{j}$ and $K_{k}$ are commuting: $K_{j} K_{k}=K_{k} K_{j}$;

(v) $I_{j}$ and $K_{k}$ are commuting: $I_{j} K_{k}=K_{k} I_{j}$, whenever $j \neq k$;

(vi) $I_{j} K_{j}=K_{j} I_{j}=0$.

Now we have

$$
\begin{aligned}
\mathbb{C}_{2 n} & =\prod_{j=1}^{n} \mathbb{C}_{2 n}\left(I_{j}+K_{j}\right) \\
& =\mathbb{C}_{2 n} I_{1} I_{2} \cdots I_{n} \oplus \mathbb{C}_{2 n} I_{1} I_{2} \cdots I_{n-1} K_{1} \oplus \ldots \oplus \mathbb{C}_{2 n} K_{1} K_{2} \cdots K_{n}
\end{aligned}
$$

obtaining in this way a direct sum decomposition of the complex Clifford algebra $\mathbb{C}_{2 n}$ into $2^{n}$ isomorphic copies of spinor space. 
We will supply all of them with a different notation, according to the specific idempotent involved:

$$
\mathbb{S}_{\left\{j_{1}, \ldots, j_{t}\right\}\left\{k_{1}, \ldots, k_{s}\right\}}=\mathbb{C}_{2 n} I_{j_{1}} I_{j_{2}} \cdots I_{j_{t}} K_{k_{1}} K_{k_{2}} \cdots K_{k_{s}}
$$

where $s+t=n,\left\{j_{1}, \ldots, j_{t}\right\} \cap\left\{k_{1}, \ldots, k_{s}\right\}=\emptyset$ and $\left\{j_{1}, \ldots, j_{t}\right\} \cup\left\{k_{1}, \ldots, k_{s}\right\}=$ $\{1, \ldots, n\}$. In what follows, we will refer to these spaces as "spinor spaces", well knowing that this is, in fact, a slight abuse of language, since there is only one spinor space, with different realizations. Each space $\mathbb{S}_{\left\{j_{1}, \ldots, j_{t}\right\}\left\{k_{1}, \ldots, k_{s}\right\}}$ has dimension $2^{n}$, its basis being obtained by right multiplation of the basis of $\mathbb{C}_{2 n}$ by the corresponding idempotent $I_{j_{1}} \cdots I_{j_{t}} K_{k_{1}} \cdots K_{k_{s}}$. In this way an alternative basis for the complex Clifford algebra $\mathbb{C}_{2 n}$ is obtained, see Figure 2 for the case $n=2$.

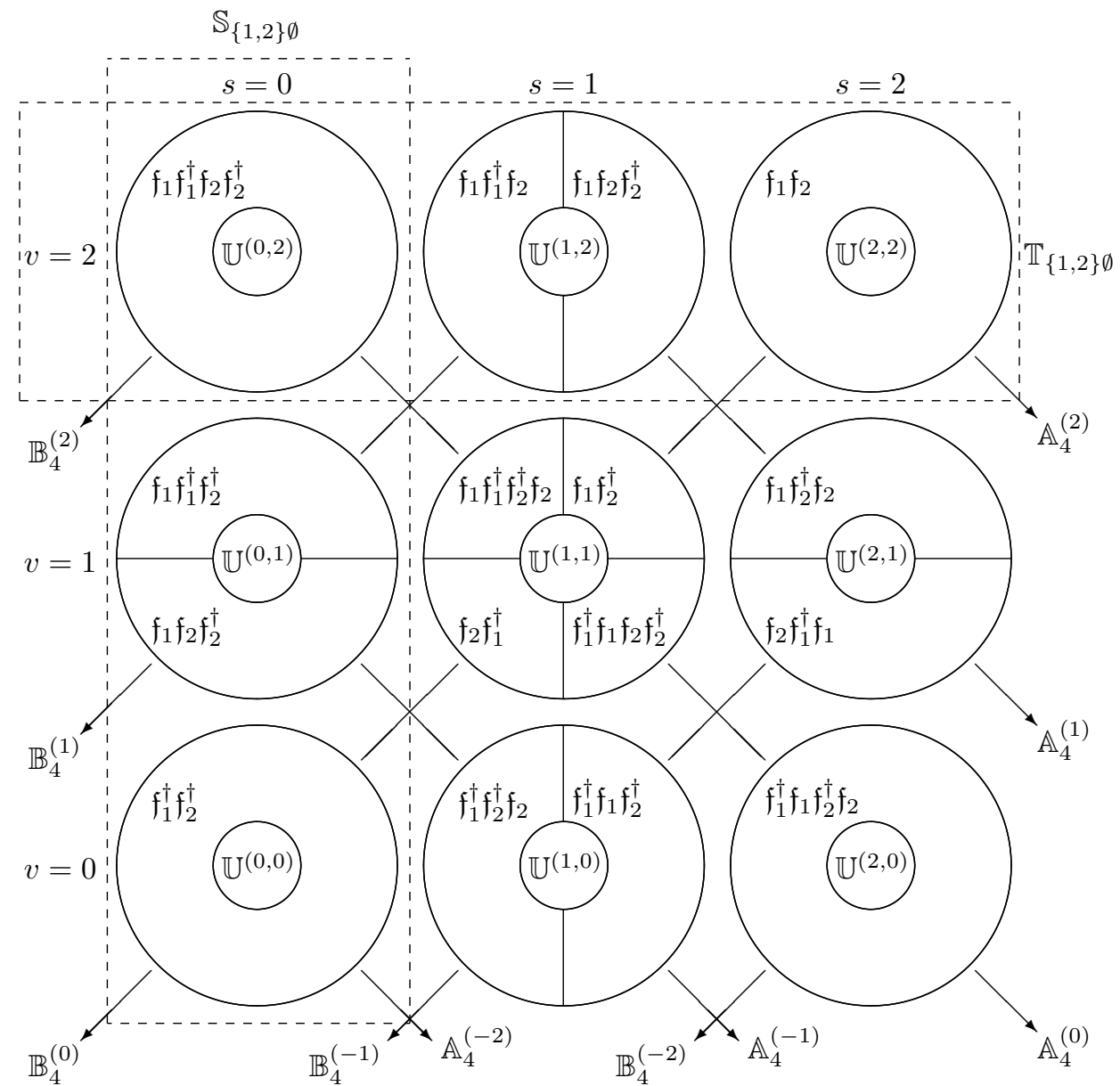

Figure 2. Spinor splitting of the complex Clifford algebra $\mathbb{C}_{4}$ 
An important operator is the so-called spin-Euler multiplication operator $\beta$ given by

$\beta=\sum_{j=1}^{n} K_{j}=\sum_{j=1}^{n} \mathfrak{f}_{j}^{\dagger} \mathfrak{f}_{j}=\sum_{j=1}^{n} \mathfrak{f}_{j}^{\dagger} \bullet \mathfrak{f}_{j}+\mathfrak{f}_{j}^{\dagger} \wedge \mathfrak{f}_{j}=\frac{n}{2}+\sum_{j=1}^{n} \mathfrak{f}_{j}^{\dagger} \wedge \mathfrak{f}_{j}=\frac{n}{2}+\frac{i}{2} \sum_{j=1}^{n} e_{j} e_{n+j}$

with complex conjugate

$\beta^{c}=\sum_{j=1}^{n} I_{j}=\sum_{j=1}^{n} \mathfrak{f}_{j} \mathfrak{f}_{j}^{\dagger}=\sum_{j=1}^{n} \mathfrak{f}_{j} \bullet \mathfrak{f}_{j}^{\dagger}+\mathfrak{f}_{j} \wedge \mathfrak{f}_{j}^{\dagger}=\frac{n}{2}+\sum_{j=1}^{n} \mathfrak{f}_{j} \wedge \mathfrak{f}_{j}^{\dagger}=\frac{n}{2}-\frac{i}{2} \sum_{j=1}^{n} e_{j} e_{n+j}$

Traditionally one also introduces the operator $\omega$ by putting

$$
\omega=\frac{1}{2 i} \sum_{j=1}^{n} e_{j} e_{n+j}
$$

so that

$$
\beta=\frac{n}{2}-\omega, \quad \beta^{c}=\frac{n}{2}+\omega=n-\beta
$$

This operator $\omega$ is the so-called fundamental form of the Kählerian metric, derived from the Kählerian potential; for more details we refer the reader to e.g. [22], [11].

Lemma 3.2. The spinor space $\mathbb{S}_{\left\{j_{1}, \ldots, j_{t}\right\}\left\{k_{1}, \ldots, k_{s}\right\}}$ consists of eigenvectors with eigenvalue $s$ for the multiplication operator $\beta$ acting from the right.

\section{Proof}

Take $\sigma \in \mathbb{S}_{\left\{j_{1}, \ldots, j_{t}\right\}\left\{k_{1}, \ldots, k_{s}\right\}}$, then $\sigma$ takes the form

$$
\sigma=\lambda I_{j_{1}} \cdots I_{j_{t}} K_{k_{1}} \cdots K_{k_{s}}, \quad \lambda \in \mathbb{C}_{2 n}
$$

Multiplication on the right with $\beta=\sum_{i=1}^{n} \mathfrak{f}_{i}^{\dagger} \mathfrak{f}_{i}=\sum_{i=1}^{n} K_{i}$ yields

$$
\sigma \beta=\sum_{i=1}^{n} \sigma K_{i}=\sum_{i=1}^{n} \lambda I_{j_{1}} \cdots I_{j_{t}} K_{k_{1}} \cdots K_{k_{s}} K_{i}
$$

where $\sigma K_{i}=0$ when $i \in\left\{j_{1}, \ldots, j_{t}\right\}$, while $\sigma K_{i}=\sigma$ when $i \in\left\{k_{1}, \ldots, k_{s}\right\}$, from which it follows that $\sigma \beta=s \sigma$.

Corollary 3.3. For each $s \in\{0,1, \ldots, n\}$ fixed, there are $\left(\begin{array}{l}n \\ s\end{array}\right)$ spinor spaces with eigenvalue $s$ for the right multiplication with $\beta$, and so their direct sum

$$
\mathbb{S}^{(s)}=\bigoplus \mathbb{S}_{\left\{j_{1}, \ldots, j_{t}\right\}\left\{k_{1}, \ldots, k_{s}\right\}}
$$

is the corresponding eigenspace for the right action of $\beta$, the sum being taken over all possible subsets $\left\{k_{1}, \ldots, k_{s}\right\}$ of $\{1, \ldots, n\}$.

In a similar way multiplication of the duality relations with $\mathbb{C}_{2 n}$ from the right yields an alternative direct sum decomposition of the complex Clifford algebra, viz

$$
\mathbb{C}_{2 n}=I_{1} I_{2} \cdots I_{n} \mathbb{C}_{2 n} \oplus I_{1} I_{2} \cdots I_{n-1} K_{1} \mathbb{C}_{2 n} \oplus \ldots \oplus K_{1} K_{2} \cdots K_{n} \mathbb{C}_{2 n}
$$


into the following $2^{n}$ isomorphic spaces of dimension $2^{n}$ :

$$
\mathbb{T}_{\left\{j_{1}, \ldots, j_{v}\right\}\left\{k_{1}, \ldots, k_{u}\right\}}=I_{j_{1}} I_{j_{2}} \cdots I_{j_{v}} K_{k_{1}} K_{k_{2}} \cdots K_{k_{u}} \mathbb{C}_{2 n}
$$

where $u+v=n,\left\{j_{1}, \ldots, j_{v}\right\} \cap\left\{k_{1}, \ldots, k_{u}\right\}=\emptyset$ and $\left\{j_{1}, \ldots, j_{v}\right\} \cup\left\{k_{1}, \ldots, k_{u}\right\}=$ $\{1, \ldots, n\}$. We will call these spaces dual spinor spaces. Quite naturally we have the following results similar to the above.

Lemma 3.4. The dual spinor space $\mathbb{T}_{\left\{j_{1}, \ldots, j_{v}\right\}\left\{k_{1}, \ldots, k_{u}\right\}}$ consists of eigenvectors with eigenvalue $v$ for the multiplication operator $\beta^{c}$ acting from the left.

Corollary 3.5. For each $v \in\{0,1, \ldots, n\}$ fixed, there are $\left(\begin{array}{l}n \\ v\end{array}\right)$ dual spinor spaces with eigenvalue $v$ for the left multiplication with $\beta^{c}$, and so their direct sum

$$
\mathbb{T}^{(v)}=\bigoplus \mathbb{T}_{\left\{j_{1}, \ldots, j_{v}\right\}\left\{k_{1}, \ldots, k_{u}\right\}}
$$

is the corresponding eigenspace for the left action of $\beta^{c}$, the sum being taken over all possible subsets $\left\{j_{1}, \ldots, j_{v}\right\}$ of $\{1, \ldots, n\}$.

So it becomes clear that each spinor space may be decomposed into subspaces each of which has a specific $v$-eigenvalue, $v$ running from 0 to $n$; we call these subspaces the homogeneous parts of the considered spinor space and we put

$$
\mathbb{S}_{\left\{j_{1}, \ldots, j_{t}\right\}\left\{k_{1}, \ldots, k_{s}\right\}}^{(v)}=\mathbb{S}_{\left\{j_{1}, \ldots, j_{t}\right\}\left\{k_{1}, \ldots, k_{s}\right\}} \cap \mathbb{T}^{(v)}, \quad v=0,1, \ldots, n
$$

In a similar way the homogeneous parts of a dual spinor space are the subspaces with a particular value of the $s$-eigenvalue, $s$ running from 0 to $n$, and we put

$$
\mathbb{T}_{\left\{j_{1}, \ldots, j_{v}\right\}\left\{k_{1}, \ldots, k_{u}\right\}}^{(s)}=\mathbb{T}_{\left\{j_{1}, \ldots, j_{v}\right\}\left\{k_{1}, \ldots, k_{u}\right\}} \cap \mathbb{S}^{(s)}, \quad s=0,1, \ldots, n
$$

The decomposition of the complex Clifford algebra $\mathbb{C}_{2 n}$ into a square of (dual) spinor spaces and their homogeneous parts is particularly suited for illustrating the action of the isotropic (left and right) multiplication operators $\mathfrak{f}_{j}$ and $\mathfrak{f}_{j}^{\dagger},(j=1, \ldots, n)$. Under left multiplication they act vertically, upwards for $\mathfrak{f}_{j}$ and downwards for $\mathfrak{f}_{j}^{\dagger}$. Under right multiplication they act horizontally, to the right for $\mathfrak{f}_{j}$ and to the left for $\mathfrak{f}_{j}^{\dagger}$. This means that left multipication with the Witt basis vectors leaves the spinor spaces invariant, while multiplication from the right leaves the dual spinor spaces invariant, which is, of course, also clear from the corresponding definitions. For each spinor and each dual spinor space this leads to the exact sequences

$$
\begin{aligned}
& 0 \overleftarrow{\mathfrak{f}_{j}^{\dagger}} \mathbb{S}_{\left\{j_{1}, \ldots, j_{t}\right\}\left\{k_{1}, \ldots, k_{s}\right\}}^{(0)} \underset{\mathfrak{f}_{j}^{\dagger}}{\stackrel{\mathfrak{f}_{j}}{\rightleftarrows}} \mathbb{S}_{\left\{j_{1}, \ldots, j_{t}\right\}\left\{k_{1}, \ldots, k_{s}\right\}}^{(1)} \stackrel{\cdots}{\underset{\ldots}{\rightleftarrows}} \mathbb{S}_{\left\{j_{1}, \ldots, j_{t}\right\}\left\{k_{1}, \ldots, k_{s}\right\}}^{(n)} \stackrel{\mathfrak{f}_{j}}{\longrightarrow} 0 \\
& 0 \overleftarrow{\mathrm{f}_{j}^{\dagger}} \mathbb{T}_{\left\{j_{1}, \ldots, j_{v}\right\}\left\{k_{1}, \ldots, k_{u}\right\}}^{(0)} \underset{\mathrm{f}_{j}^{\dagger}}{\stackrel{\mathfrak{f}_{j}}{\rightleftarrows}} \mathbb{T}_{\left\{j_{1}, \ldots, j_{v}\right\}\left\{k_{1}, \ldots, k_{u}\right\}}^{(1)} \stackrel{\cdots}{\underset{\ldots}{\rightleftarrows}} \mathbb{T}_{\left\{j_{1}, \ldots, j_{v}\right\}\left\{k_{1}, \ldots, k_{u}\right\}}^{(n)} \stackrel{\mathfrak{f}_{j}}{\longrightarrow} 0
\end{aligned}
$$

for left and right multiplication, respectively. It is important to remark here that both complexes preserve the (dual) spinor spaces as well. 
A coarser decomposition of $\mathbb{C}_{2 n}$ is obtained by considering the $(n+1)^{2}$ subspaces

$$
\mathbb{U}^{(s, v)}=\mathbb{S}^{(s)} \cap \mathbb{T}^{(v)}
$$

each of which is characterized by the pair of eigenvalues $(s, v)$, leading to

$$
\mathbb{C}_{2 n}=\bigoplus_{s=0}^{n} \bigoplus_{v=0}^{n} \mathbb{U}^{(s, v)}
$$

(see Figure 2 for the case $n=2$ ). Note that the column corresponding to the eigenvalue $s$ for right multiplication with $\beta$ contains $\left(\begin{array}{l}n \\ s\end{array}\right)$ spinor spaces $\mathbb{S}_{\left\{j_{1}, \ldots, j_{t}\right\}\left\{k_{1}, \ldots, k_{s}\right\}}$ as subcolumns, while the row corresponding to the eigenvalue $v$ for left multiplication with $\beta^{c}$ contains $\left(\begin{array}{l}n \\ v\end{array}\right)$ dual spinor spaces $\mathbb{T}_{\left\{j_{1}, \ldots, j_{v}\right\}\left\{k_{1}, \ldots, k_{u}\right\}}$ as subrows.

\section{The $\mathcal{J}$-spaces}

Following [21] we introduce the $\mathcal{J}$-operator as follows.

Definition 4.1. For an arbitrary complex Clifford number $\lambda \in \mathbb{C}_{2 n}$ one puts

$$
\mathcal{J}[\lambda]=[\omega, \lambda]=\omega \lambda-\lambda \omega
$$

Note that alternative expressions for the action of $\mathcal{J}$ are given by

$$
\mathcal{J}[\lambda]=\left[\beta^{c}, \lambda\right]=[\lambda, \beta]=\beta^{c} \lambda+\lambda \beta-n \lambda
$$

Lemma 4.2. The $\mathcal{J}$-operator acts as a derivative.

Proof

For arbitrary complex Clifford numbers $\lambda, \mu \in \mathbb{C}_{2 n}$ one has

$$
\mathcal{J}[\lambda \mu]=\omega \lambda \mu-\lambda \mu \omega=\omega \lambda \mu-\lambda \omega \mu+\lambda \omega \mu-\lambda \mu \omega=\mathcal{J}[\lambda] \mu+\lambda \mathcal{J}[\mu]
$$

In the following lemma and proposition we investigate the action of $\mathcal{J}$ on the basis vectors of the Grassmann subspace $\bigwedge_{2 n}^{p, q}$.

Lemma 4.3. For all $j=1, \ldots, n$ one has $\mathcal{J}\left[\mathfrak{f}_{j}\right]=\mathfrak{f}_{j}$ and $\mathcal{J}\left[\mathfrak{f}_{j}^{\dagger}\right]=-\mathfrak{f}_{j}^{\dagger}$.

Proposition 4.4. For any basis vector in $\bigwedge_{2 n}^{p, q}$ one has

$$
\mathcal{J}\left[\mathfrak{f}_{j_{1}}^{\dagger} \wedge \cdots \wedge \mathfrak{f}_{j_{p}}^{\dagger} \wedge \mathfrak{f}_{k_{1}} \wedge \cdots \wedge \mathfrak{f}_{k_{q}}\right]=(q-p) \mathfrak{f}_{j_{1}}^{\dagger} \wedge \cdots \wedge \mathfrak{f}_{j_{p}}^{\dagger} \wedge \mathfrak{f}_{k_{1}} \wedge \cdots \wedge \mathfrak{f}_{k_{q}}
$$

\section{Proof}

First we express the wedge products in terms of the Clifford product:

$$
\begin{aligned}
\mathcal{J}\left[\mathfrak{f}_{j_{1}}^{\dagger}\right. & \left.\wedge \cdots \wedge \mathfrak{f}_{j_{p}}^{\dagger} \wedge \mathfrak{f}_{k_{1}} \wedge \cdots \wedge \mathfrak{f}_{k_{q}}\right] \\
& =\mathcal{J}\left[\mathfrak{f}_{j_{1}}^{\dagger} \cdots \mathfrak{f}_{j_{p}}^{\dagger} \wedge \mathfrak{f}_{k_{1}} \cdots \mathfrak{f}_{k_{q}}\right] \\
& =\mathcal{J}\left[\frac{1}{2}\left(\mathfrak{f}_{j_{1}}^{\dagger} \cdots \mathfrak{f}_{j_{p}}^{\dagger} \mathfrak{f}_{k_{1}} \cdots \mathfrak{f}_{k_{q}}+(-1)^{p+q-1} \mathfrak{f}_{k_{1}} \cdots \mathfrak{f}_{k_{q}} \mathfrak{f}_{j_{1}}^{\dagger} \cdots \mathfrak{f}_{j_{p}}^{\dagger}\right)\right]
\end{aligned}
$$


Now using Lemmata 4.2-4.3 we obtain for the first term

$$
\begin{aligned}
\mathcal{J}\left[\mathfrak{f}_{j_{1}}^{\dagger} \cdots \mathfrak{f}_{j_{p}}^{\dagger} \mathfrak{f}_{k_{1}} \cdots \mathfrak{f}_{k_{q}}\right]= & \mathcal{J}\left[\mathfrak{f}_{j_{1}}^{\dagger}\right] \mathfrak{f}_{j_{2}}^{\dagger} \cdots \mathfrak{f}_{j_{p}}^{\dagger} \mathfrak{f}_{k_{1}} \cdots \mathfrak{f}_{k_{q}}+\mathfrak{f}_{j_{1}}^{\dagger} \mathcal{J}\left[\mathfrak{f}_{j_{2}}^{\dagger} \cdots \mathfrak{f}_{j_{p}}^{\dagger} \mathfrak{f}_{k_{1}} \cdots \mathfrak{f}_{k_{q}}\right] \\
= & -\mathfrak{f}_{j_{1}}^{\dagger} \mathfrak{f}_{j_{2}}^{\dagger} \cdots \mathfrak{f}_{j_{p}}^{\dagger} \mathfrak{f}_{k_{1}} \cdots \mathfrak{f}_{k_{q}}+\mathfrak{f}_{j_{1}}^{\dagger} \mathcal{J}\left[\mathfrak{f}_{j_{2}}^{\dagger}\right] \mathfrak{f}_{j_{3}}^{\dagger} \cdots \mathfrak{f}_{j_{p}}^{\dagger} \mathfrak{f}_{k_{1}} \cdots \mathfrak{f}_{k_{q}} \\
& +\mathfrak{f}_{j_{1}}^{\dagger} \mathfrak{f}_{j_{2}}^{\dagger} \mathcal{J}\left[\mathfrak{f}_{j_{3}}^{\dagger} \cdots \mathfrak{f}_{j_{p}}^{\dagger} \mathfrak{f}_{k_{1}} \cdots \mathfrak{f}_{k_{q}}\right]
\end{aligned}
$$

Continuing in the same way we are lead to

$$
\mathcal{J}\left[\mathfrak{f}_{j_{1}}^{\dagger} \cdots \mathfrak{f}_{j_{p}}^{\dagger} \mathfrak{f}_{k_{1}} \cdots \mathfrak{f}_{k_{q}}\right]=(q-p) \mathfrak{f}_{j_{1}}^{\dagger} \cdots \mathfrak{f}_{j_{p}}^{\dagger} \mathfrak{f}_{k_{1}} \cdots \mathfrak{f}_{k_{q}}
$$

Also for the second term we obtain, in a similar way

$$
\mathcal{J}\left[\mathfrak{f}_{k_{1}} \cdots \mathfrak{f}_{k_{q}} \mathfrak{f}_{j_{1}}^{\dagger} \cdots \mathfrak{f}_{j_{p}}^{\dagger}\right]=(q-p) \mathfrak{f}_{k_{1}} \cdots \mathfrak{f}_{k_{q}} \mathfrak{f}_{j_{1}}^{\dagger} \cdots \mathfrak{f}_{j_{p}}^{\dagger}
$$

Adding both terms the desired result folows.

Hence $\bigwedge_{2 n}^{p, q}$ consists of eigenvectors of the operator $\mathcal{J}$ with eigenvalue $j=q-p$ and so the direct sum

$$
\mathbb{C}_{2 n}^{(j)}=\bigoplus_{q-p=j} \bigwedge_{2 n}^{p, q}
$$

is the corresponding eigenspace. The eigenspaces $\mathbb{C}_{2 n}^{(j)}$ are found on the $(2 n+1)$ diagonals $q-p=j, j=-n, \ldots, n$ in the square Grassmann decomposition of $\mathbb{C}_{2 n}$ (see Figure 1 for the case $n=2$ ).

We may also investigate the action of the operator $\mathcal{J}$ on the $\mathbb{U}^{(s, v)}$-cells of the (dual) spinor decomposition of $\mathbb{C}_{2 n}$. Take a Clifford number $\mu$ in such a cell $\mathbb{U}^{(s, v)}=\mathbb{S}^{(v)} \cap \mathbb{T}^{(s)}$, then

$$
\mathcal{J}[\mu]=\beta^{c} \mu+\mu \beta-n \mu=v \mu+s \mu-n \mu=(v+s-n) \mu
$$

This means that the $\mathbb{U}^{(s, v)}$-cells consist of eigenvectors of the operator $\mathcal{J}$ with eigenvalue $j=v+s-n$ ( $j$ running from $-n$ to $n$ ), and so the direct sums

$$
\mathbb{A}_{2 n}^{(j)}=\bigoplus_{v+s=j+n} \mathbb{U}^{(s, v)} \quad, \quad j=-n, \ldots, n
$$

are the corresponding eigenspaces. These $\mathcal{J}$-eigenspaces $\mathbb{A}_{2 n}^{(j)}$ are to be found on the $(2 n+1)$ anti-diagonals $v+s=j+n,(j=-n, \ldots, n)$ in the square decomposition of $\mathbb{C}_{2 n}$ into (dual) spinor spaces (see Figure 2 for the case $n=2$ ).

So we have found that $\mathbb{C}_{2 n}^{(j)}$ and $\mathbb{A}_{2 n}^{(j)}$ both are eigenspaces of the operator $\mathcal{J}$ with the same eigenvalue, whence they coincide: for each $j=-n, \ldots, n$ one has

$$
\mathbb{C}_{2 n}^{(j)}=\bigoplus_{q-p=j} \bigwedge_{2 n}^{p, q}=\bigoplus_{v+s=j+n} \mathbb{U}^{(s, v)}=\mathbb{A}_{2 n}^{(j)}
$$


The basis vectors of $\mathbb{A}_{2 n}^{(j)}$ may thus be reshuffled to obtain those of $\mathbb{C}_{2 n}^{(j)}$; let us illustrate this in the case where $n=2$ for the eigenvalue $j=0$ :

$$
\begin{aligned}
\mathbb{A}_{4}^{(0)}=\operatorname{span}_{\mathbb{C}}\left(\mathfrak{f}_{1} \mathfrak{f}_{1}^{\dagger} \mathfrak{f}_{2} \mathfrak{f}_{2}^{\dagger}, \mathfrak{f}_{1} \mathfrak{f}_{1}^{\dagger} \mathfrak{f}_{2}^{\dagger} \mathfrak{f}_{2}, \mathfrak{f}_{1}^{\dagger} \mathfrak{f}_{1} \mathfrak{f}_{2} \mathfrak{f}_{2}^{\dagger}, \mathfrak{f}_{1}^{\dagger} \mathfrak{f}_{1} \mathfrak{f}_{2}^{\dagger} \mathfrak{f}_{2}, \mathfrak{f}_{1} \mathfrak{f}_{2}^{\dagger}, \mathfrak{f}_{2} \mathfrak{f}_{1}^{\dagger}\right) \\
=\operatorname{span}_{\mathbb{C}}\left(\frac{1}{4}+\frac{1}{2} \mathfrak{f}_{2} \wedge \mathfrak{f}_{2}^{\dagger}+\frac{1}{2} \mathfrak{f}_{1} \wedge \mathfrak{f}_{1}^{\dagger}+\mathfrak{f}_{1} \wedge \mathfrak{f}_{1}^{\dagger} \wedge \mathfrak{f}_{2} \wedge \mathfrak{f}_{2}^{\dagger},\right. \\
\frac{1}{4}+\frac{1}{2} \mathfrak{f}_{2}^{\dagger} \wedge \mathfrak{f}_{2}+\frac{1}{2} \mathfrak{f}_{1} \wedge \mathfrak{f}_{1}^{\dagger}+\mathfrak{f}_{1} \wedge \mathfrak{f}_{1}^{\dagger} \wedge \mathfrak{f}_{2}^{\dagger} \wedge \mathfrak{f}_{2}, \\
\frac{1}{4}+\frac{1}{2} \mathfrak{f}_{2} \wedge \mathfrak{f}_{2}^{\dagger}+\frac{1}{2} \mathfrak{f}_{1}^{\dagger} \wedge \mathfrak{f}_{1}+\mathfrak{f}_{1}^{\dagger} \wedge \mathfrak{f}_{1} \wedge \mathfrak{f}_{2} \wedge \mathfrak{f}_{2}^{\dagger}, \\
\left.\frac{1}{4}+\frac{1}{2} \mathfrak{f}_{2}^{\dagger} \wedge \mathfrak{f}_{2}+\frac{1}{2} \mathfrak{f}_{1}^{\dagger} \wedge \mathfrak{f}_{1}+\mathfrak{f}_{1}^{\dagger} \wedge \mathfrak{f}_{1} \wedge \mathfrak{f}_{2}^{\dagger} \wedge \mathfrak{f}_{2}, \mathfrak{f}_{1} \wedge \mathfrak{f}_{2}^{\dagger}, \mathfrak{f}_{2} \wedge \mathfrak{f}_{1}^{\dagger}\right) \\
=\operatorname{span}_{\mathbb{C}}\left(1, \mathfrak{f}_{1}^{\dagger} \wedge \mathfrak{f}_{1}, \mathfrak{f}_{2}^{\dagger} \wedge \mathfrak{f}_{2}, \mathfrak{f}_{1}^{\dagger} \wedge \mathfrak{f}_{2}, \mathfrak{f}_{1} \wedge \mathfrak{f}_{2}^{\dagger}, \mathfrak{f}_{1}^{\dagger} \wedge \mathfrak{f}_{2}^{\dagger} \wedge \mathfrak{f}_{1} \wedge \mathfrak{f}_{2}\right)=\mathbb{C}_{4}^{(0)}
\end{aligned}
$$

\section{The $\mathcal{K}$-spaces}

Again following [21] we now introduce the $\mathcal{K}$-operator.

Definition 5.1. For an arbitrary complex Clifford number $\lambda \in \mathbb{C}_{2 n}$ one puts

$$
\mathcal{K}[\lambda]=\{\omega, \lambda\}=\omega \lambda+\lambda \omega
$$

Note that alternative expressions for the action of $\mathcal{K}$ are given by

$$
\mathcal{K}[\lambda]=\beta^{c} \lambda-\lambda \beta=n \lambda-\{\beta, \lambda\}=\left\{\beta^{c}, \lambda\right\}-n \lambda
$$

Proposition 5.2. For arbitrary complex Clifford numbers $\lambda, \mu \in \mathbb{C}_{2 n}$ one has

$$
\mathcal{K}[\lambda \mu]=\mathcal{K}[\lambda] \mu-\lambda \mathcal{J}[\mu]=\mathcal{J}[\lambda] \mu+\lambda \mathcal{K}[\mu]
$$

\section{Proof}

We consecutively have

$$
\begin{aligned}
\mathcal{K}[\lambda \mu]=\omega \lambda \mu+\lambda \mu \omega & =\omega \lambda \mu+\lambda \omega \mu-\lambda \omega \mu+\lambda \mu \omega=\mathcal{K}[\lambda] \mu-\lambda \mathcal{J}[\mu] \\
& =\omega \lambda \mu-\lambda \omega \mu+\lambda \omega \mu+\lambda \mu \omega=\mathcal{J}[\lambda] \mu+\lambda \mathcal{K} \mu
\end{aligned}
$$

In order to identify the eigenspaces of the operator $\mathcal{K}$ we again consider a complex Clifford number $\mu$ in the cell $\mathbb{U}^{(s, v)}$. We obtain

$$
\mathcal{K}[\mu]=\beta^{c} \mu-\mu \beta=v \mu-s \mu=(v-s) \mu
$$

meaning that the $\mathbb{U}^{(s, v)}$-cells consist of eigenvectors of the operator $\mathcal{K}$ with eigenvalue $k=v-s \quad(k$ running from $-n$ to $n)$, and so the direct sums

$$
\mathbb{B}_{2 n}^{(k)}=\bigoplus_{v-s=k} \mathbb{U}^{(s, v)} \quad, \quad k=-n, \ldots, n
$$


are the corresponding eigenspaces. These $\mathcal{K}$-eigenspaces $\mathbb{B}_{2 n}^{(k)}$ are to be found on the $(2 n+1)$ diagonals $v-s=k$ in the square decomposition of $\mathbb{C}_{2 n}$ into (dual) spinor spaces (see Figure 2 for the case $n=2$ ). Now it also follows that

Proposition 5.3. The operators $\mathcal{J}$ and $\mathcal{K}$ commute.

Proof

It suffices to decompose any complex Clifford number into its $\mathbb{U}^{(s, v)}$-components, and to observe that the operators $\mathcal{J}$ and $\mathcal{K}$ commute on these $\mathbb{U}^{(s, v)}$-cells.

\section{Merging the $\mathbb{S}$-, $\mathbb{T}$-, $\mathcal{J}$ - and $\mathcal{K}$-spaces}

In Section 4 we have seen that starting with the Grassmann subspaces $\bigwedge_{2 n}^{p, q}$, $(0 \leq p, q \leq n)$, we can collect them according to constant $(q-p)$-value to form eigenspaces $\mathbb{C}_{2 n}^{(j)}$ with eigenvalue $j=q-p$ for the operator $\mathcal{J}$. Each eigenspace $\mathbb{C}_{2 n}^{(j)}$ coincides with a specific direct sum of homogeneous parts of different spinor and dual spinor spaces:

$$
\mathbb{C}_{2 n}^{(j)}=\mathbb{A}_{2 n}^{(j)}=\bigoplus_{v+s=j+n} \mathbb{U}^{(s, v)}
$$

each of these $\mathbb{U}^{(s, v)}$-cells consisting of eigenvectors of the operator $\mathcal{K}$ with eigenvalue $k=v-s$. Hence each $\mathbb{C}_{2 n}^{(j)}$ may still be split up further into eigenspaces of the $\mathcal{K}$ operator, whence we put for $j=-n, \ldots, n$ :

$$
\mathbb{C}_{2 n}^{(j, k)}=\mathbb{A}_{2 n}^{(j)} \cap \mathbb{B}_{2 n}^{(k)}=\left\{\mu \in \mathbb{C}_{2 n}^{(j)}: \mathcal{K}[\mu]=k \mu\right\}
$$

or

$$
\begin{aligned}
\mathbb{C}_{2 n}^{(j, k)} & =\left\{\mu \in \mathbb{C}_{2 n}: \mathcal{J}[\mu]=j \mu \text { and } \mathcal{K}[\mu]=k \mu\right\} \\
& =\left\{\mu \in \mathbb{C}_{2 n}: \omega \mu=\frac{j+k}{2} \mu \text { and } \mu \omega=\frac{j-k}{2} \mu\right\}
\end{aligned}
$$

Note that these $\mathbb{C}_{2 n}^{(j, k)}$-cells are precisely Michelsohn's $\mathbf{C} \ell^{\mathbf{p}, \mathbf{q}}$ subspaces (see [21]) after the identification $j=p$ and $k=q$. Note also that in each $\mathbb{C}_{2 n}^{(j)}$ with $j$ fixed, the $\mathcal{K}$-eigenvalues are running from $|j|-n$ to $-|j|+n$ with step 2 since $k=v-s=j+n-2 s, s=0,1, \ldots, n$ and so

$$
\mathbb{C}_{2 n}^{(j)}=\bigoplus_{\substack{k=|j|-n \\ \text { step2 }}}^{-|j|+n} \mathbb{C}_{2 n}^{(j, k)}
$$

Similarly in each $\mathbb{C}_{2 n}^{(j)}$ with $k$ fixed, the $\mathcal{J}$-eigenvalues are running from $|k|-n$ to $-|k|+n$ with step 2 since $j=v+s-n=k-n+2 s, s=0,1, \ldots, n$. In this way 
another square decomposition of the complex Clifford algebra $\mathbb{C}_{2 n}$ is obtained, into simultaneous eigenspaces of the operators $\mathcal{J}$ and $\mathcal{K}$ :

$$
\mathbb{C}_{2 n}=\bigoplus_{j=-n}^{n} \bigoplus_{\substack{k=|j|-n \\ \text { step2 }}}^{-|j|+n} \mathbb{C}_{2 n}^{(j, k)}=\bigoplus_{k=-n}^{n} \bigoplus_{\substack{j=|k|-n \\ \text { step2 }}}^{-|k|+n} \mathbb{C}_{2 n}^{(j, k)}
$$

However take $\mu \in \mathbb{U}^{(s, v)}$, then $\mathcal{J}[\mu]=(v+s-n) \mu$ and $\mathcal{K}[\mu]=(v-s) \mu$, which means $j=v+s-n$ and $k=v-s$, from which it follows that $s=\frac{1}{2}(n+j-k)$ and $v=\frac{1}{2}(n+j+k)$, and vice versa. We thus have shown that in terms of the eigenvalues of the $\mathcal{J}$ and $\mathcal{K}$ operators:

$$
\mathbb{A}_{2 n}^{(j)} \cap \mathbb{B}_{2 n}^{(k)}=\mathbb{C}_{2 n}^{(j, k)}=\mathbb{U}^{\left(\frac{n+j-k}{2}, \frac{n+j+k}{2}\right)}=\mathbb{S}^{\left(\frac{n+j-k}{2}\right)} \cap \mathbb{T}^{\left(\frac{n+j+k}{2}\right)}
$$

or, equivalently, in terms of the eigenvalues of the $\beta$ operator:

$$
\mathbb{S}^{(s)} \cap \mathbb{T}^{(v)}=\mathbb{U}^{(s, v)}=\mathbb{C}_{2 n}^{(v+s-n, v-s)}=\mathbb{A}_{2 n}^{(v+s-n)} \cap \mathbb{B}_{2 n}^{(v-s)}
$$

This means that both patchworks of the complex Clifford algebra $\mathbb{C}_{2 n}$ consisting either of (dual) spinor spaces and their homogeneous parts or of $\mathcal{J}$ - and $\mathcal{K}$-eigenspaces, coincide, but the former are running horizontally and vertically while the latter are running (anti-)diagonally.

\section{Acknowledgements}

This research is part of a joint project of the Clifford Research Group at Ghent University and the Mathematical Institute of Charles University Prague, supported by the bilateral scientific co-operation between Flanders and Czech Republic. V. Souček acknowledges support by the institutional grant MSM 0021620839 and by grant GA CR 201/08/0397. F. Brackx and H. De Schepper acknowledge support by the institutional grant BOF06/BIL/012.

\section{References}

[1] F. Brackx , J. Bureš, H. De Schepper, D. Eelbode, F. Sommen and V. Souček, Fundaments of Hermitean Clifford Analysis. Part I: Complex structure, Compl. Anal. Oper. Theory 1(3) (2007), 341-365.

[2] F. Brackx, J. Bureš, H. De Schepper, D. Eelbode, F. Sommen and V. Souček, Fundaments of Hermitean Clifford Analysis. Part II: Splitting of h-monogenic equations, Complex Var. Elliptic Eq. 52(10-11) (2007), 1063-1079.

[3] F. Brackx, B. De Knock and H. De Schepper, A matrix Hilbert transform in Hermitean Clifford analysis, J. Math. Anal. Appl. 344 (2008), 1068-1078.

[4] F. Brackx, B. De Knock, H. De Schepper and F. Sommen, On Cauchy and MartinelliBochner integral formulae in Hermitean Clifford analysis, Bull. Braz. Math. Soc. New Series 40(3) (2009) 395-416.

[5] F. Brackx, R. Delanghe and F. Sommen, Clifford Analysis, Pitman Publishers, Boston-London-Melbourne, 1982. 
[6] F. Brackx, R. Delanghe and F. Sommen, Differential forms and/or multivector functions, Cubo 7(2) (2005) 139-169.

[7] F. Brackx, H. De Schepper, D. Eelbode and V. Souček, The Howe dual pair in Hermitean Clifford analysis, to appear in Rev. Mat. Iberoamericana.

[8] F. Brackx, H. De Schepper, R. Lávička and V. Souček, Dirac and Moisil-Téodorescu systems in Hermitean Clifford Analysis (to appear).

[9] F. Brackx, H. De Schepper and F. Sommen, A theoretical framework for wavelet analysis in a Hermitean Clifford setting, Comm. Pure Appl. Anal. 6(3), 2007, 549567.

[10] F. Brackx, H. De Schepper and F. Sommen, The Hermitean Clifford analysis toolbox, Appl. Clifford Algebras 18(3-4) (2008), 451-487.

[11] F. Brackx, H. De Schepper and V. Souček, Differential Forms versus Multivector Functions in Hermitean Clifford Analysis, to appear in Cubo.

[12] F. Brackx, H. De Schepper and V. Souček, Hermitean Clifford Analysis on Kählerian manifolds (to appear).

[13] A. Damiano, D. Eelbode and I. Sabadini, Invariant syzygies for the Hermitian Dirac operator, Math. Zeitschrift 262 (2009), 929-945.

[14] R. Delanghe, R. Lávička and V. Souček, On polynomial solutions of generalized Moisil-Théodoresco systems and Hodge-de Rham systems (to appear).

[15] R. Delanghe, R. Lávička and V. Souček, The Fischer decomposition for Hodge-de Rham systems in Euclidean spaces (to appear).

[16] R. Delanghe, F. Sommen and V. Souček, Clifford Algebra and Spinor-Valued Functions, Kluwer Academic Publishers, Dordrecht - Boston - London, 1992

[17] J. Gilbert and M. Murray, Clifford Algebras and Dirac Operators in Harmonic Analysis, Cambridge University Press, Cambridge, 1991.

[18] K. Gürlebeck, K. Habetha and W. Sprößig, Holomorphic Functions in the Plane and n-dimensional Space, Birkhäuser Verlag, Basel, 2008.

[19] R. Lavicka, On the structure of monogenic multivector valued polynomials. In: T.E. Tsimos, G. Psihoyios, Ch. Tsitouras (eds.), Proceedings of ICNAAM 2009, AIP Conference Proceedings 1168, 793-796.

[20] R. Lavicka, The Fischer decomposition for the H-action and its applications, submitted for Proceedings of ISAAC 2009, London.

[21] M.L. Michelsohn, Clifford and Spinor Cohomology of Kähler Manifolds, American Journal of Mathematics 102(6) (1980), 1083-1146.

[22] A. Moroianu, Lectures on Kähler geometry, London Mathematical Society Student Texts 69, Cambridge University Press, Cambridge, 2007.

[23] I.R. Porteous, Topological Geometry, Van Nostrand Reinhold Company, London New York - Toronto - Melbourne, 1969

[24] I. Sabadini and F. Sommen, Hermitian Clifford analysis and resolutions, Math. Meth. Appl. Sci. 25 (16-18) (2002), 1395-1414. 
F. Brackx

Clifford Research Group, Faculty of Engineering, Ghent University, Galglaan 2, 9000

Gent, Belgium

e-mail: freddy.brackx@ugent.be

H. De Schepper

Clifford Research Group, Faculty of Engineering, Ghent University, Galglaan 2, 9000 Gent, Belgium

e-mail: hennie.deschepper@ugent.be

V. Souček

Mathematical Institute, Faculty of Mathematics and Physics, Charles University, Sokolovská 83, 18675 Praha, Czech Republic

e-mail: soucek@karlin.mff.cuni.cz 